journal club

\title{
Desmoteplase in einem Zeitfenster von 3 bis 9 Stunden nicht wirksamer als Placebo
}

Fragestellung: Die Studie ging der Frage nach, ob Desmoteplase, eine Substanz zur systemischen Thrombolyse, bei Patienten mit schweren Schlaganfällen in einem Zeitfenster von 3 bis 9 Stunden wirksam ist.

Hintergrund: Die klassische zugelassene Therapie des akuten ischämischen Insults ist eine Behandlung mit Gewebeplasminogenaktivator (rt-PA) in einem Zeitfenster bis zu 4,5 Stunden. Desmoteplase hat eine deutlich längere Halbwertszeit und könnte daher in einem längeren Zeitfenster nach Beginn der neurologischen Ausfälle wirksam sein.

In den Dosisfindungsstudien DIAS, DEDAS und DIAS-2 ergaben sich Hinweise darauf, dass Desmoteplase möglicherweise bei Patienten mit Verschlüssen der großen proximalen Hirnarterien wirksam sein könnte [1]. Vor diesem Hintergrund wurden deshalb zwei größere randomisierte Studien, nämlich DIAS-3 und DIAS-4 initiiert, die eine Dosis von $90 \mu \mathrm{g} / \mathrm{kg}$ Körpergewicht Desmoteplase in einem Zeitfenster zwischen 3 und 9 Stunden untersuchten. Die DIAS-3-Studie wurde im November 2013 beendet und zeigte keinen Nutzen von Desmoteplase [2].

von Kummer R, Mori E, Truelsen T et al. Desmoteplase 3 to 9 hours after major artery occlusion stroke: The DIAS-4 Trial (Efficacy and Safety Study of Desmoteplase to Treat Acute Ischemic Stroke). Stroke 2016; 47: $2880-7$
Patienten und Methodik: Die DIAS-4-Studie schloss Patienten mit ischämischen Schlaganfall und einem in der CT- oder MR-Angiografie nachgewiesenen Verschluss oder einer hochgradigen Stenose der großen hirnversorgenden Arterien ein. Die Patienten erhielten entweder $90 \mu \mathrm{g} / \mathrm{kg}$ Desmoteplase oder Placebo. Der primäre Endpunkt war ein Wert auf der modifizierten Rankin-Skala (mRS) von 0 bis 2 nach 90 Tagen. Im Rahmen der Sicherheitsanalyse wurden Sterblichkeit, symptomatische intrakranielle Blutungen und andere schwerwiegende unerwünschte Arzneimittelwirkungen erfasst.

Ergebnisse: Die DIAS-4-Studie wurde nach dem Bekanntwerden der Ergebnisse von DIAS-3 abgebrochen. Bis zu diesem Zeitpunkt hatte man 252 DIAS-4-Patienten in die Studie eingeschlossen. In der mit Desmoteplase behandelten Gruppe erreichten $41,9 \%$ einen mRS von 0 bis 2 und in der Placebogruppe $36 \%$. Dieser Unterschied war statistisch nicht signifikant.

Die Sterblichkeit, die Häufigkeit intrakranieller Blutungen und andere schwerwiegende unerwünschte Arzneimittelereignisse waren in beiden Gruppen identisch. In einer gepoolten Analyse von DIAS-3, DIAS-4 und einer japanischen Studie (DIAS-J) erreichten 184 von 376 mit Desmoteplase behandelte Patienten, entsprechend 48,9\%, einen Score von 0 bis 2 auf der modifizierten Rankin-Skala verglichen mit 171 von 381 Patienten $(44,9 \%)$ in der Placebogruppe. Die Odds Ratio betrug 1,33, der Unterschied zwischen den beiden Behandlungsgruppen war nicht signifikant. Die Rekanalisierungsrate betrug $49,3 \%$ bei Behandlung mit Desmoteplase versus $38,3 \%$ bei Placebobehandlung und war mit einer Odds Ratio von 1,59 signifikant höher in der mit Desmoteplase behandelten Gruppe.

Schlussfolgerungen: Eine Behandlung mit Desmoteplase in einem Zeitfenster von 3 bis 9 Stunden bei schweren Schlaganfällen mit Verschlüssen der proximalen Hirnarterien ist einer Behandlung mit Placebo nicht überlegen.

\section{- Kommentar von Hans-Christoph Diener, Essen}

\section{Desmoteplase hat keine Zukunft in der Schlaganfalltherapie}

Die beiden Zulassungsstudien DIAS-3 und DIAS-4 haben leider die Ergebnisse der Dosisfindungsstudien bestätigt. Desmoteplase ist in einem Zeitfenster von 3 bis 9 Stunden nicht wirksam, auch wenn nur Patienten eingeschlossen werden, bei denen ein proximaler Gefäßverschluss bildgebend nachgewiesen wurde. Desmoteplase führte zwar zu einer höheren Rekanalisierungsrate als Placebo, verbesserte aber nicht den funktionellen Outcome. Damit dürfte die Entwicklung von Desmoteplase zur Schlaganfalltherapie ihr Ende gefunden haben.

Referenzen:

1. Von Kummer R et al. Int J Stroke 2012; 7: 589-96

2. Albers GW et al. Lancet Neurol 2015; 14: $575-84$

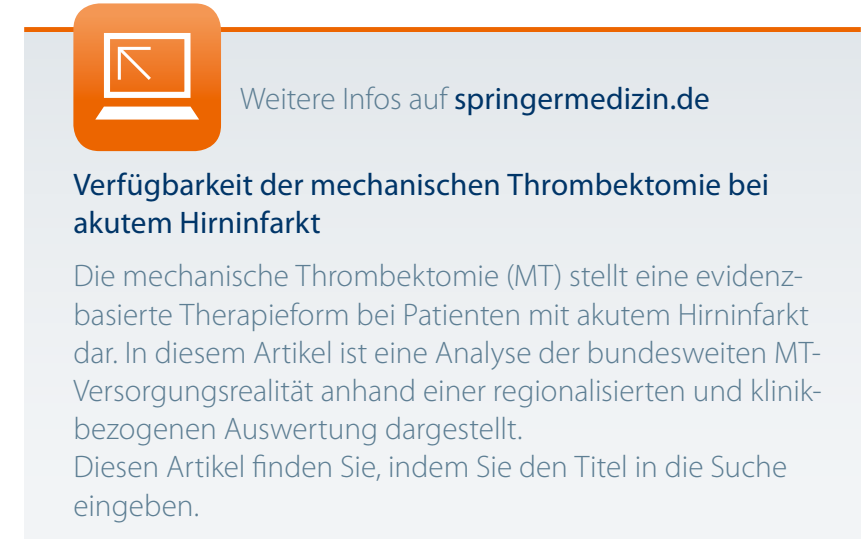

